\title{
Treatment of glossopharyngeal neuralgia by carbamazepine
}

\author{
R SAVIOLO, G FIASCONARO \\ From Divisione di Cardiologia, Ospedale Mauriziano "Umberto $I^{\circ ", ~ T u r i n, ~ I t a l y ~}$
}

SUMMARY An elderly woman who presented with Adams-Stokes attacks that were preceded by paroxysms of neuralgia was successfully treated with carbamazepine. Ventricular asystole was provoked by pharyngeal pain and coincided with loss of consciousness. Vagal reflexes are probably responsible for the bradycardia that causes the cardiac symptoms of glossopharyngeal neuralgia. Carbamazepine (600 mg/day) abolished the pharyngeal pain and associated cardiovascular manifestations in this patient for at least ten months.

Glossopharyngeal neuralgia is a rare cause of syncope. Neuralgia in the throat and neck triggers severe bradycardia up to asystole, and this leads to cardiac syncope, sometimes with seizures. ${ }^{12}$ We report the case of an elderly woman who had syncopal attacks preceded by paroxysms of neuralgia. This patient was successfully treated with carbamazepine (Tegretol).

\section{Case report}

An 83 year old woman was admitted in October 1985 because of Adams-Stokes attacks, which were preceded by excruciating pain in the left ear and pharynx. Pain was provoked by swallowing and head movements. She had had typhoid fever as an infant and glomerulonephritis as a young adult. She reported episodes of chest pain over the past ten years that subsided with sublingual glyceryl trinitrate; these were managed as sporadic angina. She was admitted to the coronary care unit where an abrupt slowing down of the heart rate to 20-25 beats/minute with concomitant loss of consciousness and convulsive movements were noted during continuous electrocardiographic monitoring. A temporary transvenous pacemaker was inserted to prevent prolonged asystole and syncope. A few hours later a sharp pain in the left ear and throat was precipitated by coughing. This was followed by asystole, which was interrupted by pacemaker intervention. She had a 3/6 systolic ejection murmur that was loudest at the

Requests for reprints to Dr R Saviolo, Divisione di Cardiologia, Ospedale Mauriziano "Umberto $I^{\circ}$ ", Corso Turati 62, 10128 Turin, Italy. fourth left parasternal intercostal space and radiated to the neck. This murmur was compatible with mild aortic stenosis. There were no abnormal neurological signs. Blood chemistry was within normal limits, except for a haemoglobin concentration of $10 \mathrm{mg} / \mathrm{dl}$ and slightly increased blood glucose and urea nitrogen.

Chest $x$ ray showed cardiomegaly, aortic calcification, and pulmonary emphysema. Electroencephalography and computed tomography of the brain were normal. The electrocardiogram showed sinus bradycardia ( 42 beats/minute), anterior hemiblock, and inverted T waves in III and a VF. Echocardiographic findings were compatible with calcific aortic stenosis of moderate degree and good left ventricular systolic function. The results of endoscopy of the upper gastrointestinal tract were normal.

The clinical picture led to a diagnosis of cardiac syncope due to glossopharyngeal neuralgia. To confirm the diagnosis, the patient was asked to drink carbonated water. This provoked pharyngeal pain and, almost immediately, ventricular asystole $(5.0 \mathrm{~s})$, accompanied by feeling lightheaded. Carotid sinus massage did not produce pain or severe bradycardia. On the other hand, after the pacemaker was switched off, intravenous atropine sulphate $(2.0 \mathrm{mg})$ prevented ventricular asystole despite continuing pharyngeal pain.

Because she had a history of chest pain, sympathomimetic agents, such as isoprenaline, were not tried. When the patient was started on carbamazepine (Tegretol) $600 \mathrm{mg} /$ day there was a prompt symptomatic improvement. There were no major side effects. The temporary pacemaker was then removed because it was regarded as unneces- 


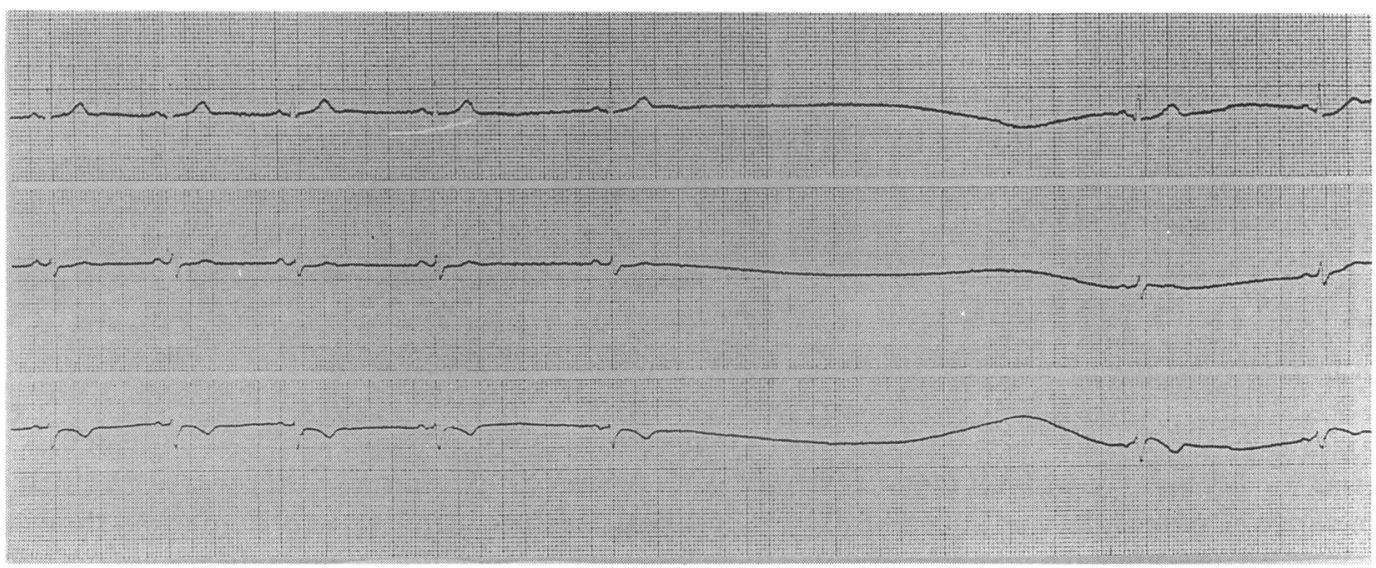

Figure Electrocardiogram (leads I, II, III (from top to bottom); paper speed $25 \mathrm{~mm} / \mathrm{s}$ ) showing sinus arrest lasting $4 \cdot 8 \mathrm{~s}$ after pharyngeal pain evoked by drinking carbonated water.

sary in the absence of recurrent syncope. Ten months after treatment was started the patient remains symptom free on carbamazepine.

\section{Discussion}

Glossopharyngeal neuralgia was first described by Weisenburg in 1910 in a patient with a tumour of the cerebello-pontine angle. ${ }^{3}$ Riley et al in 1942 were the first to report a cardiac arrest associated with neuralgia. ${ }^{4}$ The cause of this disorder was usually undetermined, though in a few reported cases cerebello-pontine angle tumours ${ }^{1}$ or an elongated styloid process or a calcified stylohyoid ligament or both $^{5}$ were identified as aetiological factors. These may cause chronic compression of nerves during swallowing. Some cases improved with surgical resection of the anomalous process. ${ }^{5}$

It is likely that vagal reflexes, initiated by glossopharyngeal neuralgia, are involved in the arrhythmias most commonly found in this syndrome, that is, sinus arrest, sinus bradycardia, and wandering atrial pacemaker. ${ }^{6}$ Hypersensitivity of the carotid sinus is not a factor in glossopharyngeal neuralgia because massage of the sinuses does not reproduce the typical cardiovascular signs. ${ }^{12}$ The paroxysms of pain are triggered by swallowing, particularly cold, salted, bitter, or acid foods ${ }^{26}$ and by mechanical stimulation of the tonsillar fossa on the affected side (trigger zone).

Treatment aims at abolishing dysphagia and the ensuing bradycardia-syncope reflex. Atropine and isoprenaline, together with a temporary pacemaker, are used as emergency measures, but are not suitable for long term treatment because they do not relieve the neuralgia. Local anaesthesia of the pharynx and nerve block with mepivacaine (carbocaine) are additional measures that produce temporary benefit. ${ }^{26}$ Anticonvulsant treatment (phenytoin) was advo- cated as the long term treatment of choice. ${ }^{7}$ However, the clinical response of glossopharyngeal neuralgia to phenytoin was variable. ${ }^{6-8}$ Carbamazepine is an effective treatment of trigeminal neuralgia, and may also be an alternative to phenytoin in the medical management of glossopharyngeal neuralgia. In our case carbamazepine was effective at relatively low dosage $(600 \mathrm{mg} /$ day or $10 \mathrm{mg} / \mathrm{kg} /$ day) in abolishing paroxysms of pain and associated cardiovascular manifestations for at least ten months without any side effects. Our findings indicate that carbamazepine may be an alternative to surgical resection of the glossopharyngeal nerve, which remains the most effective cure.

\section{References}

1 Garretson HD, Elvidge AR. Glossopharyngeal neuralgia with asystole and seizures. Arch Neurol 1963;8:26-31.

2 Kong Y, Heyman A, Entman ML, et al. Glossopharyngeal neuralgia associated with bradycardia, syncope and seizures. Circulation 1964;30:109-13.

3 Weisenburg TH. Cerebello-pontine tumor diagnosed for six years as tic douleureux; the symptoms of irritation of the ninth and twelfth cranial nerves. JAMA 1910;54:1600-4.

4 Riley HA, German WJ, Wortis H, et al. Glossopharyngeal neuralgia initiating or associated with cardiac arrest. Trans Am Neurol Assoc 1942;68:28-9.

5 Graf CJ. Glossopharyngeal neuralgia and ossification of the stylohyoid ligament. J Neurosurg 1959;16:448-53.

6 Khero BA, Mullins CB. Cardiac syncope due to glossopharyngeal neuralgia. Arch Intern Med 1971;128:806-8.

7 Jannone A, Baker AB, Morrell F. Dilantin in the treatment of trigeminal neuralgia. Neurology 1958;8:126-8.

8 Kersley JA. A case of glossopharyngeal neuralgia. $J$ Laryngol Otol 1965;79:734-7. 